\title{
Comedo Necrosis
}

National Cancer Institute

\section{Source}

National Cancer Institute. Comedo Necrosis. NCI Thesaurus. Code C36128.

A morphologic finding that indicates the presence of a cancerous tumor with a central area that contains necrotic cancer cells. 